\title{
Diuretics increase Blood Creatinine in the Treatment of Hypertension
}

\author{
Marwa F. Elmahdy, Noor M. Allehyani, Nabawia M. Shehata, Maryam O. Alanazi \\ ${ }^{1}$ Clinical Pathologist and Lecturer of Clinical Biochemistry at Laboratory Department, Applied Medical Sciences \\ College, Shaqra University. KSA, ${ }^{2}$ Lecturer at Laboratory Department, Applied Medical Sciences College, Shaqra \\ University. KSA, ${ }^{3}$ Assistant Professor at Laboratory Department, Applied Medical Sciences College, Shaqra \\ University. KSA, ${ }^{4}$ Lecturer at laboratory Department, Applied Medical Sciences College, Shaqra University. KSA
}

\begin{abstract}
Background: Diuretics have been used in the treatment of hypertension by either alone or combining it with other antihypertensive drugs. It treated about $50 \%$ of hypertensive patients alone and about $80 \%$ when treatment was a combination with other antihypertensive drugs. Oral diuretics are used as an initiating antihypertensive treatment in stepped care approach the antihypertensive management .One such loop diuretic called as Furosemide is being regularly used in different stages of acute kidney injury and hypertension. Lowering the blood pressure is ultimately reducing the risk of cardiovascular morbidity and mortality. The sideeffects of diuretics are dose dependent. The intake of Furosemide will bring an increase in the creatinine level.It must be noted that if the diuretics are been taken for prolong usage, it might over-stress the function of kidney which will result in sodium resorption increase and elevation of creatinine levels.
\end{abstract}

Material and Method: Twenty hypertensive patients in the age range of 40 to 76 years old were subjected to a treatment with furosemide $(20 \mathrm{mg} /$ day) for a variable period of one to three years. The analysis of blood samples was being done against the serum creatinine following the colorimetric method. The results were statistically processed and analyzed by SPSS using the T-test method.

Results: The trial was runon the participants being either hypertensive patients or normal individuals. The Mean (+/-SD) of age was $59.4+/-10.3$, mean (+/-SD) of duration of treatment with furosemide was $2.1+/-$ 0.7 . Mean $(+/-\mathrm{SD})$ of serum creatinine in the control individual was $0.9+/-0.22$, and mean serum creatinine in hypertensive patients treated with furosemide was $2.37+/-0.69$. ( $\mathrm{P}$ value $<0.05$.)

Conclusion: The diuretic Furosemide can increase thelevel of creatinine.

Aim of the work: Determine the effect of diuretics (furosemide) on serum creatinine.

Keywords: Diuretics, creatinine, Hypertension.

\section{Introduction}

Diuretics are being commonly usedand being claimed as safe antihypertensive drugs over the experience ofnumerous decades of clinical application. Diuretics represent a better clinical outcome for the hypertensive patients ${ }^{1}$.

Diuretics are used to correct thecomposition and the volume of body fluids, which treat hypertension. There are different types of diuretics: a) The most common antihypertensive drugs called thiazides and thiazide-like agents which help in inhibiting sodium re-absorption in the early distal convoluted tubule b) The loop diuretics like furosemide which inhibit $\mathrm{Na} / \mathrm{K} / 2 \mathrm{Cl}$ co-transport system in the thick ascending limb of the Henle loop c) The diuretics which can retain potassium and the aldosterone receptor blockers like epithelial sodium channel (amyloid) and spironolactone. Theyintervenein the sodium reabsorption and the excretion of hydrogen and potassium in the late distal tubule, the cortical collecting duct and the connecting tubule ${ }^{2}$.

Some diuretics as ifthiazides and loop diuretics have shown that their actions on antihypertensive groundsare 
independent of the effect they are bring in the diuretics. The reason for reduction in blood pressure cannot be accounted to decreased blood volume achieved by increasing urine production, ratherbeing affected by other mechanisms and at lower dosage than that is required to produce diuresis ${ }^{3}$.

Advantages of Diuretics: Lowering blood pressure will decrease the risk of cardiovascular clinical issues including the mortality. Also, lowering BP will minimize thehazards of stroke and heart failure. A slump in cardiovascular events was diuretics based ${ }^{4}$.

Numerous studies have proved that that diuretics protect the body from the consequences of falling into osteoporosis conditionas wellminimizes the threat of hip fractures ${ }^{5}$.

Reid et al. $2000^{6}$, claimed that hydrochlorothiazide lowered the loss of cortical bone in the women who are into postmenopausal phase. Schoofs et al. $2003^{7}$,showed that thiazide acts as a protection against hip fractures and lasts within 4 months if the usage is being stopped. Thus apart from their use inlowering blood pressure, thiazide proves to play a pivotal role in the avoidance of osteoporosis condition and fractures. Other additional therapeutic advantage of Diuretic therapy is that it can reduce the threatsof cardiovascular complications ${ }^{8}$.

The patient has been taken care well of his associated disorders which includesdiabetes mellitus,coronary related artery disease,overweight,lipid profile disorders and the metabolic syndrome. True health efficacy implies adecrease of total or cerebral- and cardiovascular mortality established by blood pressure declineusing diuretics (thiazides) and beta-blockers in lengthy period studies. They prove to work in as a sufficient replacement either in the reduction of blood pressure that has been affected by the others drugsconsumed individually or in combined mode?

Side effects of Diuretics: Diuretics, like any other anti-hypertensive drug, prone to bring in some side effects. The most noticeable ones are hypokalemia, plasma lipid profile change, glucose intolerance and hyperuricemia. The hypokalemia induced by diuretics may be hazardous when combinedwith digitalistherapy ${ }^{\mathbf{1 0}}$.

All the recommendations were being generated by a treatment approach evaluating the characteristics of patients and the preponderance of guidelinesbased on confirmation for the classes of drugsrather than drugs being consumed individually. It should be thought if a hypertensive patient could be subjected to the diuretic treatment or not ${ }^{11}$.

Diuretics remain as the first choice drugs in treating high blood pressure due to their effectiveness (particularly in blacks and the aged masses), the antihypertensive initiation efficacy of the other compounds, their little side-effects profile and their economical cost ${ }^{\mathbf{1 0}}$. Loop diuretics could be beneficial in case of chronic related kidney disease with serum creatinine greater than 1.50 milligrams/deciliter or e GF lesser than 30 milligrams/ minute/1.73 square meter ${ }^{12}$.

Their antihypertensive effect might be enhanced with the administration of drugs during the nighttime. Diuretics are being commonly usedand being claimed as safe antihypertensive drugs over the experience ofnumerousdecennium of clinical application ${ }^{\mathbf{1 3}}$.

The concept of using diuretics to achieve better clinical outcomes for patient is considered the main for clinician prescription ${ }^{11}$.

The atherosclerotic, and the changes which are related to hypertension-related vascular lesions that can be affecting in the kidney primarily is subjected to affect the pre- glomerular arterioles, they can be a wide range of variation resulting in ischemic changes which is most likely to occur in the glomeruli and probably it can be in post-glomerular structures also ${ }^{14}$.Glomerular injury can occur and maybe a probable stage in which the damage is done to the glomerular capillaries, which can be considered by the basic reason due to glomerular hyperactive perfusion.

Glomerular pathology can be further used to investigate the progresses to glomerulosclerosis, however, it can also provide a proper resulting diagnosis of renal tubules that may be responsible andmay become ischemic finally subjecting to a change in gradually atrophic. The renal lesion which is supposed to be associated with malignant hypertension is more likely to contain fibrinoid necrosis which is indirectly connected to the afferent arterioles, sometimes it can be found extending into the glomerulus, which can result in the increase of focal necrosis of the glomerular tuft. So, it can be well identified that hypertension is a risk factor which may result factor for renal injury and can account for the end-stage renal disease ${ }^{\mathbf{1 5}}$.

As per the study that is given, the long-term use of 
diuretics can also be considered well associated with the increased incidence that is responsible of renal cell carcinoma. Grossman et al.,1999 16 ,the right to a conclusion that $55 \%$ of the patients who are treated by the diuretics for over long periods of renal cell carcinoma are more in number then in patients who are subjected to be treated with diuretics compared with the diuretic nonusers. The renal tubular cells, which are considered the major areas of effect with the diuretics, many considered one of the origin of malignancy.

Furosemide (Lasix) can be considered one of the ingredients that can cause elevations of BUN. This type of reversible elevations can be caused and are associated with the dehydration of the, and as a result, they should be avoided in the patients with the acute symptoms for the renal insufficiency ${ }^{17}$.

Uremia is considered a state in which hyperactive responsiveness can be seen in the patient, which can thereby lead to the infection rates as very high in the populations, which are undergoing dialysis ${ }^{\mathbf{1 8}}$.It can be thus deduced that the direct effect of the diuretic drugs can become a main cause for the increase in the mortality for the patient's renal function. It can also further lead to the nonrecovery of the functions which can lead to a delay in the institution of renal support (it can be caused with the overload of the dialysis volume, or it can be with reference to the reversible predicted azotemia), or there can be various other unknown factors that may be a cause for the situation ${ }^{19}$.

Diuretics can also be one of the reasons which is able to increase the level of creatinine in the blood of the patient. Yet another causes that can be associated with the long-term use of diuretics drugs can be the overwork of the kidney in order to compensate and increase in sodium resorption, and this is a primary cause which can increase very high creatinine levels. By diuretic-use, there can be a considerable decrease in the volume of the plasma as well as the sodium level. Thus in order to maintain the compensation of the situation, it is really necessary to constrict the efferent arteriole. This mechanism will help to preserve glomerular filtration rate (GFR). However GFR is not assumed to be really increasing. The fraction that is filtered can be found increasing part.

This is due to the decrease in the flow of the renal plasma. Hence, it can be deduced from the above conditions that if the plasma filtered is less from glomerulus per unit time, then the production of the serum creatinine must increase to compensate the variation. However, there are certain Western medicines that are responsible for increasing the level of creatinine. But these medicines are subjected to provide side effects which cannot be ignored easily in the kidney treatment. However, the patients are subjected to know that best high creatinine level treatment can be a really great help to make kidneys work again rapidly. Once the kidney start functioning properly, it will be used for the removal of the waste and the various harmful substances that are available in the blood, which may reduce creatinine level naturally ${ }^{\mathbf{2 0}}$.

Loop diuretics are the most common mechanism, which is used for the control of congestive heart attack symptoms, but it can be considered that these agents are actually responsible for various neuro hormonal activations. This type of activations can worsen the situation for the survival. It should be noted that blood urea nitrogen (BUN)levels increase with neurohormonal activation ${ }^{\mathbf{2 1}}$.

There is a very strong risk which is associated to the use of the higher levels of diuretic drugs which can depend on BUN concentrations. There is a very strong chance for the reduced survival in the patients with elevated BUN and it can be considered that there are furthermore, better chances for survival in the patients with normal BUN. The information and the data which is available in the report supported at the reference tends to provide a very clear role for neuro-hormonal activation that can be caused as a result in high dose loop diuretic associated mortality. ${ }^{21}$.

\section{Material and Method}

These patients were 20 aged between 40 -76 years old, they were hypertensive and treated with furosemide(20mg/day) for (1-3) year's period and their blood samples were analyzed against serum creatinine by colorimetric method. The results are analyzed by SPSS using T-test.

- All the patients are examined by measuring blood pressure and estimate their creatinine blood level.

- Blood samples are collected from patients and serum samples were obtained after centrifuged at $2000 \mathrm{rpm}$ for 10 minutes.

- Serum creatinine is estimated by kineticJaff's reactionwithout deproteinization method 22 . 


\section{Results}

Table (1): Patients Data

\begin{tabular}{|l|c|c|}
\hline & Range & Mean \pm S.D. \\
\hline Age & $40-76$ & $59.4 \pm 10.3$ \\
\hline Duration of treatment & $1-3$ & $2.1 \pm 0.7$ \\
\hline
\end{tabular}

Table (2): Serum creatinine level in patients treated with furosemide.

\begin{tabular}{|l|c|c|}
\hline Creatinine (mg\%) & Disease & Control \\
\hline Range & $1.3-3.5$ & $0.6-1.2$ \\
\hline Mean \pm SD & $2.37 \pm 0.69$ & $0.90 \pm 0.22$ \\
\hline T. test & \multicolumn{2}{|c|}{4.605} \\
\hline P. value & \multicolumn{2}{|c|}{$0.001 *$} \\
\hline
\end{tabular}

Result Analysis: The trial was run on participant either hypertensive patients or normal individuals. Mean (SD) age was $59.4+/-10.3$, mean (SD) of duration of treatment with furosemide was $2.1+/-0.7$. Mean (SD) of serum creatinine in control individual was $0.9+/-0.22$, and mean serum creatinine in hypertensive patients treated with furosemide was $2.37+/-0.69$. (P value $<$ 0.05.).

\section{Discussion}

Furosemideis considered to be a loop diuretic, and have the main functionality in the increasing of the output of Eugene in the patients with acute injuries in the kidney ${ }^{23}$. This drug is also utilized for the modification of the volume as well. With the increase in the composition of the fluid in the body of the patient, very high chances are available for the treatment of hypertension ${ }^{2}$.

The treatment can be done with the variable and very high doses of the drug furosemide, which makes it possible for the conversion of the oliguric to nonoliguric acute renal insufficiency. This conversion makes it possible as an induction effect for harmful oxidative stress that is found inside the kidneys. A randomized controlled trial is required for the powered and controlled diagnosis of the drug to yield the clinical benefits of this dosing strategy. It can be helpful for the modification of the potential harms that can be caused and subjected to this diagnosis ${ }^{23}$.

The results that have been approved in the study in this research proves that there was an increase in the serum creatinine level for all the patients who were treated with the drug furosemide (those who were hypertensive patients), if the group is compared to a controlled nature population. $(\mathrm{P}<0.05)$ this disagree with Tiziana proved thatintermittent furosemide administration that is reflected on the population of the patients is considered to be not associated with the increased issues in the patients. There is no associative increase in the mortality of the patients who are suffering from hypertension or wire at the risk for kidney injuries that are cute in nature. However, it could be understood that the reduction of mortality can be one of the reasons that can be undertaken as a preventive measure for the treatment ${ }^{24}$.

On one hand, it can also be identified with various studies that diuretics can decrease the senior nature of the acute renal failure, which will be helpful enough for the transformation of oliguric to not oliguric. It is also worth noticing that the duration for the acute kidney injuries can be reduced considerably by the improvement in the speed for the recovery of the renal functions and thus, overall, it will be carefully helpful to minimize or reduce the actual need of renal replacements. As an additional advantage,furosemide can also be proved very helpful for the management of the increase in the volume as well as the improvement in the electrolyte homeostasis, which can be considered to be actually related with the acute kidney injury results ${ }^{25,26}$.

Our results agree with Mehat ${ }^{19}$, who showed that using of diuretics for the patience that are critically in a state and unstable condition having acute kidney injuries may not be able to associate with the improvement for the clinical outcomes and there can be reasons that probably increase the mortality of such population ${ }^{27}$.

Our result are also in Association with the findings with Sean et al., Where it can be very easily shown that furosemide did not reduce the rate of worsening in kidney diseases. This in turn can be one of the important factors that proves that the drug is not associated for abnormalities that are caused by increased electrolytes ${ }^{28}$.

Ethical Clearance: The researchers were able to asked permission to conduct the study through the deanship of scientific research and manage to request official permission from Manger of universal Hospital in Shaqra, Government University in KSA to select the samples and data collection. The researchers explained the aim of the study to the respondents and confidentiality. 
Source of Funding: There was no fund produced from any organization.

Conflict of Interest: There is no conflict of interest.

\section{References}

1. Boukhris M, Abcha F, Elhadj ZI, Kachboura SJIhj. Which diuretic for which hypertensive patient? 2017;69(2):282.

2. Padilla MCA, Armas-Hernández MJ, Hernández RH, Israili ZH, Valasco MJAjot. Update of diuretics in the treatment of hypertension. 2007;14(2):15460 .

3. Cadwallader $\mathrm{AB}$, De La Torre X, Tieri A, Botrè FJBjop. The abuse of diuretics as performanceenhancing drugs and masking agents in sport doping: pharmacology, toxicology and analysis. 2010;161(1):1-16.

4. Liu L, Wang Z, Gong L, Zhang Y, Thijs L, Staessen $\mathrm{JA}$, et al. Blood pressure reduction for the secondary prevention of stroke: a Chinese trial and a systematic review of the literature. 2009;32(11):1032-40.

5. Bolland M, Ames R, Horne A, Orr-Walker B, Gamble G, Reid IJOi. The effect of treatment with a thiazide diuretic for 4 years on bone density in normal postmenopausal women. 2007;18(4):47986.

6. Reid IR, Ames RW, Orr-Walker BJ, Clearwater JM, Horne AM, Evans MC, et al. Hydrochlorothiazide reduces loss of cortical bone in normal postmenopausal women: a randomized controlled trial. 2000;109(5):362-70.

7. Schoofs MW, van der Klift M, Hofman A, de Laet CE, Herings RM, Stijnen T, et al. Thiazide diuretics and the risk for hip fracture. 2003;139(6):476-82.

8. Uzu T, Kimura GJC. Diuretics shift circadian rhythm of blood pressure from nondipper to dipper in essential hypertension. 1999;100(15):1635-8.

9. Maisch B, Brilla C, Kruse T, Noll B, Bethge CJH. Retrospective studies and prospects of therapy for hypertension. 1995;20(6):370-89.

10. Papademetriou V. (1992): Diuretics in hypertension: clinical experiences. Eur Heart J.;13 Suppl G:92-5.

11. Prichard B, Owens C, Woolf AJEhj. Adverse reactions to diuretics. 1992;13(suppl_G):96-103.

12. Mancia GJEHJ. ESH/ESC guidelines for the management of arterial hypertension: the Task Force for the Management of Arterial5. Mancia
G, Fagard R, Narkiewicz K, et al. 2013 ESH/ ESC guidelines for the management of arterial hypertension: the Task Force for the Management of Arterial Hypertension of the European Society of Hypertension (ESH) and of the European Society of Cardiology (ESC). 2013;34(28):2159-219.

13. Hermida RC, Ayala DE, Mojon A, Chayan L, Domínguez MJ, Fontao MJ, et al. Comparison of the effects on ambulatory blood pressure of awakening versus bedtime administration of torasemide in essential hypertension. 2008;25(6):950-70.

14. Ono H and Ono Y.(1997):"Nephrosclerosis and Hypertension" The Medical Clinics of North America.,81:1273-88

15. Krzesinski J-M, Cohen EPJACB. Hypertension and the kidney. 2007;62(1):5-14.

16. Grossman E, Messerli FH, Goldbourt UJTAjoc. Does diuretic therapy increase the risk of renal cell carcinoma? 1999;83(7):1090-3.

17. Sanofi-Aventis, Revised August 2011

18. Foley RN. (2008):Infectious complications in chronic dialysis patients. Perit Dial Int.;28:S167-71

19. Mehta RL,Pascual MT,Soroko S, Chertow GM.(2002): Diuretics, mortality, and nonrecovery of renal function in acute renal failure. JAMA.;288:2547-53

20. http://www.kidneyfailureweb.com/2014

21. Testani JM, Shannon RP, Kimmel S. Interaction between Loop Diuretic Associated Mortality and Serum Blood Urea Nitrogen Concentration in Chronic Heart Failure: Insights from the BEST trial limited dataset. Am Heart Assoc; 2010.

22. Tietz N.W. (1986): Textbook of clinical chemistry.W.B. Philadelphia,1986,pp:1271-1281.

23. Silbert BI, Ho KM, Lipman J, Roberts JA, Corcoran TB, Morgan DJ, et al. Does furosemide increase oxidative stress in acute kidney injury? : Mary Ann Liebert, Inc. 140 Huguenot Street, 3rd Floor New Rochelle, NY 10801 USA; 2017.

24. Tiziana Bove, Alessandro Belletti, Alessandro Putzu, Simone Pappacena, Giuseppe Denaro, Giovanni Landoni1, Sean M. Bagshaw, Alberto Zangrillo(2018):Research article https://doi. org/10.1371/journal.pone.0196088, 2018

25. Teixeira C, Garzotto F, Piccinni P, Brienza N, Iannuzzi M, Gramaticopolo S. et al .,(2013): Neferologia e Cura Intensiva (NEFROINT) 
investigators. Fluid balance and urine volume are independent predictors of mortality in acute kidney injury. Crit Care; 17:R14

26. Bove T, Belletti A, Putzu A, Pappacena S, Denaro $\mathrm{G}$, Landoni $\mathrm{G}$, et al. Intermittent furosemide administration in patients with or at risk for acute kidney injury: Meta-analysis of randomized trials. 2018; 13(4):e0196088.

27. Teixeira C, Garzotto F, Piccinni P, Brienza N,
Iannuzzi M, Gramaticopolo S, et al. Fluid balance and urine volume are independent predictors of mortality in acute kidney injury. 2013;17(1):R14.

28. Grams ME, Estrella MM, Coresh J, Brower RG, Liu KD, National Heart L, et al. Fluid balance, diuretic use, and mortality in acute kidney injury. 2011; 6(5):966-73. 